\title{
Helicobacter pylori infection and type 1 diabetes mellitus in children
}

\section{Mohammad Reza Esmaeili Dooki ${ }^{1}$ Morteza Alijanpour Aghamaleki ${ }^{1,2}$ (D) $\cdot$ Negar Noushiravani ${ }^{3} \cdot$ Seyed Reza Hosseini ${ }^{4}$. Leila Moslemi ${ }^{1,5}$. Mahmoud Hajiahmadi ${ }^{1}$. Mohammad Pournasrollah ${ }^{6}$}

Received: 22 December 2018 / Accepted: 28 January 2020 / Published online: 4 February 2020

(C) Springer Nature Switzerland AG 2020

\begin{abstract}
Objectives This study aimed to investigate the association between Helicobacter pylori infection with diabetes mellitus type one and the effect of infected Helicobacter pylori on glycemic control.

Methods This case control study was conducted on children with and without type 1 diabetes mellitus (T1DM). Demographic data and gastrointestinal symptoms in both groups and glycemic control status and duration of diabetes were recorded in patients with T1DM. Stool test was done on all children to detect Helicobacter pylori antigen.

Results Sixty three children with T1DM with a mean of $10.88 \pm 2.84$ years and 105 control children with an average age $10.17 \pm$ 2.55 years $(P=0 / 09)$ were involved in this study. The frequency of Helicobacter pylori infection in patients with T1DM was 17/ $63(27 \%)$ and 25/105 (23.8\%) in control group, $(P=0 / 64)$. The frequency of bloating, epigastric pain and nausea was not significantly different between the two groups. The frequency of epigastric pain in children with diabetes with helicobacter infection was significantly higher than non-infected children with diabetes $(29.4 \%$ vs. $2.2 \%)(P=0.004)$. The mean duration of diabetes $(P=0.53)$, age diagnosis of diabetes $(P=0.09)$, fasting blood glucose $(P=0.18)$, glycosylated hemoglobin $(P=0.08)$ and the daily insulin dose $(P=0.18)$ in patients with T1DM with and without helicobacter pylori infection had not significantly different.

Conclusions There was no significant association between Helicobacter pylori infection and diabetes in children 5-15 years old, and glycemic control status was not difference in patients with T1DM with and without Helicobacter pylori infection.
\end{abstract}

Keywords Child $\cdot$ Diabetes mellitus type $1 \cdot$ Helicobacter pylori $\cdot$ Infection

Morteza Alijanpour Aghamaleki

m.alijanpour@yahoo.com

1 Non-Communicable Pediatric Diseases Research Center, Health Research Institute, Babol University of Medical Sciences, Babol, IR, Iran

2 Non-Communicable Pediatric Diseases Research Center, Amirkola Children's Hospital, No 19, Amirkola, Babol, Mazandaran Province 47317-41151 IR, Iran

3 Student Research Committee, Health Research Institute, Babol University of Medical Sciences, Babol, IR, Iran

4 Social Determinants of Health Research Center, Health Research Institute, Babol University of Medical Sciences, Babol, IR, Iran

5 Health Reproductive Research Center, Sari Branch, Islamic Azad University, Sari, IR, Iran

6 The Clinical Research Development Unit of Amirkola Children's Hospital, Babol University of Medical Sciences, Babol, IR, Iran

\section{Introduction}

Type-1 Diabetes Mellitus (T1DM) is a complex disease resulting from the interplay of genetic, epigenetic and environmental factors [1]. T1DM is the most common metabolic disorder in children and adolescents. The results of a large epidemiological study showed that the incidence of T1DM has increased by $2-5 \%$ worldwide [2]. T1DM can affect other organs too, such as the digestive system, as it starts from an early age [3]. children with Diabetes Mellitus can develop various infections due to different factors, including immune disorders, gastric motility disorders, increased number of hospital visits and gastrointestinal complaints; it therefore appears that Helicobacter pylori ( $H$. pylori) infection causes some gastrointestinal symptoms [4].

H. pylori, which inhabits various areas of the stomach and duodenum, is a Gram-negative helix-shaped bacterium. H. pylori infection leading to chronic gastritis is asymptomatic in the majority of carriers, but is considered a major risk factor 
for the development of gastric and duodenal ulcers and gastric malignancies [5].

The prevalence of $H$. pylori infection is associated with age. The overall prevalence of $H$. pylori infection is less than $10 \%$ in children from developed countries. H. pylori infection is more common in the developing world, and more than $80 \%$ of the population are infected before puberty [6]. A study in Sudan showed that $51 \%$ of children were seropositive for $H$. pylori infection [7]. The risk of acquiring $H$. pylori infection is associated with socioeconomic status, family education, overcrowding and lack of running water [8]. The effects of $H$. pylori in children include peptic ulceration, gastrointestinal malignancies, gastritis, malnutrition, anemia and digestive disorders leading to growth and physical retardation [9]. The link between T1DM and H. pylori still remains controversial. Some studies have suggested that the high prevalence of this infection in patients with T1DM [10-14] is related to the duration of diabetes, dyspepsia, age, sex, BMI, blood pressure, fasting plasma glucose (FPG) and Hemoglobin A1c $\left(\mathrm{HbA}_{1} \mathrm{c}\right)$ level $[10,11]$. H. pylori infection can lead to hyperglycemia in children with Diabetes Mellitus, and its mechanisms are unknown but are thought to include the secretion of counterregulatory hormones due to stress in addition to the production of cytokines. Cytokines can stimulate the secretion of counterregulatory hormones, and thus directly affect carbohydrate metabolism $[15,16]$. In contrast, other studies have reported that $H$. pylori infection is not associated with T1DM and there is no difference in the prevalence of $H$. pylori infection between children with Diabetes and children without Diabetes Mellitus [17-19]. Therefore, the relationship between diabetes and $H$. pylori still remains a controversial issue.

Due to the conflicting results about the relationship between $H$. pylori and diabetes in children, this study was conducted to compare the frequency of H. pylori infection in children with Diabetes and children without Diabetes Mellitus in Amirkola Children's Hospital of Babol (North of Iran) and to compare the glycemic control status between the children with Diabetes, with and without $H$. pylori infection.

\section{Materials and methods}

This study was conducted on patients with T1DM (at least 6 months after the onset of diabetes), referred to the Endocrine Clinic of Amirkola Children's Hospital of Babol in 2014-2015. All patients received basal-bolus protocol of insulins' Glargine and Asparte. They were compared to a group of children without Diabetes Mellitus selected from Babol schools through cluster sampling. The two groups were matched for age and gender.
The inclusion criteria consisted of consenting to participate in the study and the age 5-15 years; the exclusion criteria consisted of the use of antibiotics over the last month.

\section{Sample size}

Based on previous studies, the frequency of $H$. pylori infection is $79 \%$ in children with diabetes and $\% 51.2$ in those without diabetes. With a $90 \%$ statistical power at a $5 \%$ significance level, the sample size was calculated as 67 per group [20]. To enable a better comparison, the group of children without Diabetes Mellitus was considered double.

This study was approved by the Ethics Committee of Babol University of Medical Sciences under the code 1393.9. All the children or their parents signed consent forms, which included a comprehensive description of the study protocol and objectives.

\section{Examination and laboratory protocol}

All the children were examined by one physician and they and/ or their parents responded to questions regarding their demographic data and gastrointestinal symptoms in the past 2 weeks, such as nausea, bloating and abdominal pain. As for the patients with diabetes, data were obtained from the medical records and included the duration of diabetes (the time from disease diagnosis until entering the study), mean insulin doses (required average daily dose of insulin per week), last $\mathrm{HbA}_{1} \mathrm{c}$ and FPG level. If the recorded $\mathrm{HbA}_{1} \mathrm{c}$ and FPG belonged to more than 3 months ago, these factors were remeasured.

For measurement of $\mathrm{HbA}_{1} \mathrm{c}$ level, enzymatic method by Hitachi auto analyzer (manufactured by Diazine, USA) was used.

In the children without Diabetes Mellitus, a glucometer (GLUCOCARD 01_mini, ARKRAY Inc., Healthcare Pvt. Ltd., made in Japan) was used to measure the venous blood glucose in order to rule out their Diabetes Mellitus, and those with abnormal glucose levels were excluded. According to Bazmamoun et al. [14], the criteria for diabetes were FPG > $126(\mathrm{mg} / \mathrm{dl})$ and plasma glucose $>200 \mathrm{mg} / \mathrm{dl} 2 \mathrm{~h}$ after meals. The children with T1DM were divided into two subgroups; the first subgroup included "the patients with diabetes" with H.pylori vs. "the patients with diabetes" without H.pyolri; the second subgroup included "the patients with diabetes" with positive H.pylori who had epigastric pain vs. those without epigastric pain. A comparison was made between the two subgroups in terms of glycemic status and the average $\mathrm{HbA}_{1} \mathrm{c}$, daily insulin dosage and FPG.

The stool test was carried out in both groups to detect H. pylori antigens. A swab was dipped in $250 \mathrm{mg}$ of fresh stool up to $5 \mathrm{~mm}$ and slowly rotated to the sides. The stoolcontaining swab was placed in a buffer vial and shaken for a 
minute after closing its lid. The test cassette was then removed from its pouch and placed on the table. In the next step, one to two drops of the buffer were mixed with the stool and poured into the cassette, and after $10 \mathrm{~min}$ of waiting, the test result was read.

The test was considered negative with the appearance of a purple line and positive with the appearance of two purple lines.

The antigen was measured by the monoclonal antibody method using $H$. pylori stool kit (manufactured by Generic Assays, Germany).Internal procedural controls were included in the test. A colored band appearing in the control region $(\mathrm{C})$ was considered an internal positive procedural control. It confirmed sufficient volume and correct procedural technique. Sensitivity and specificity for $H$. pylori antigen test were 98.5\% and $98.1 \%$, respectively. Positive predictive value was $97.7 \%$ and negative predictive value was $98.6 \%$. All the tests were performed at the same laboratory (Amirkola Children's Hospital). The height and weight of the children were measured with a stadiometer and Seca scale (Seca, Germany) and their BMI was also calculated.

\section{Statistical analysis}

The data collected were analyzed SPSS-17 (Chicago, IL, USA). Descriptive statistics were used to describe the subjects' characteristics, and the Chi-square test or Fisher's exact test were used to independently determine the relationship between the variables. The mean $\pm \mathrm{SE}$ plus the t-test were used for the normally distributed data. $P$ values less than 0.05 were considered statistically significant.

\section{Results}

This study was conducted on 63 children with and 105 without Diabetes Mellitus (Table 1). Overall, 42 (25\%) children were infected by $H$. pylori. The prevalence of $H$. pylori

Table 1 A comparison of demographic data between the children with diabetes and children without diabetes mellitus

\begin{tabular}{llll}
\hline $\begin{array}{l}\text { Groups } \\
\text { Variable }\end{array}$ & $\begin{array}{l}\text { Children with } \\
\text { diabetes }\end{array}$ & $\begin{array}{l}\text { Children without } \\
\text { diabetes }\end{array}$ & $P$ value \\
\hline $\begin{array}{l}\text { Sex: } \mathrm{N}(\%) \\
\text { Male }\end{array}$ & $34(54)$ & $47(44.8)$ & 0.24 \\
$\begin{array}{l}\text { Female } \\
\begin{array}{l}\text { Ethnicity: } \mathrm{N}(\%) \\
\text { (Tabari) }\end{array}\end{array}$ & $29(46)$ & $58(55.2)$ & \\
$\begin{array}{l}\text { Age: } \mathrm{yr}(\mathrm{Mean} \pm \mathrm{SD}) \\
\mathrm{BMI}: \mathrm{Kg} / \mathrm{m}^{2}\end{array}$ & $10.88 \pm 2.84$ & $10.17 \pm 2.55$ & 0.09 \\
$\quad(\mathrm{Mean} \pm \mathrm{SD})$ & $18.89 \pm 3.65$ & $18.43 \pm 3.22$ & 0.39 \\
\hline
\end{tabular}

${ }^{\mathrm{a}} B M I$ Body mass index infection was $17(27 \%)$ in the children with Diabetes Mellitus and 25 (23.8\%) in the control group. There were no significant differences between the two groups $(P=0.64)$. The prevalence of $H$. pylori infection was not different between the two age groups of $5-10$ and $10-15$ years $(21.4 \%$ vs. $28.6 \%$, $P=0.28)$.

Clinical data including epigastric pain, flatulence and nausea were observed in ten $(9.5 \%$ vs. $3.8 \%, P=0.17)$, three $(0$ vs. $1.8 \%, P=0.29)$ and one ( 0 vs. $1 \%, P=1)$ of the children in both groups, respectively.

The children with T1DM were divided into two groups based on $H$. pylori infection: positive: $27 \%(n=17)$ and negative: $73 \%(n=46)$. Moreover, $23.5 \%$ of the 5-10 age group and $76.5 \%$ of the 10-15 age group were infected by $H$. pylori $(P=0.04)$. Age at the time of diabetes diagnosis was $8.84 \pm$ 2.03 years in the $H$. pylori positive group and $7.45 \pm 2.9$ years in the $H$. pylori negative group $(P=0.07)$; and the average duration of diabetes was $2.74 \pm 1.62$ years in the $H$. pylori positive group and $3.16 \pm 2.57$ years in the $H$. pylori negative group $(P=0.09)$. The average $\mathrm{HbA}_{1} \mathrm{c}$ measured was $8.08 \pm$ $1.51(\%)$ in the H. pylori positive group and $9.08 \pm 1.87(\%)$ in the $H$. pylori negative group $(P=0.08)$ : the average daily insulin dosage was $28.2 \pm 24.06$ units in the $H$. pylori positive group and $21.58 \pm 13.94$ units in the $H$. pylori negative group $(P=0.18)$; the average FPG was $168.17 \pm 72.28 \mathrm{mg} / \mathrm{dl}(9 / 33$ $\pm 4 \mathrm{mmol} / \mathrm{l})$ in the $H$. pylori positive group and $198.7 \pm 81.61$ $(11 \pm 4.5 \mathrm{mmol} / \mathrm{l})$ in the $H$. pylori negative group $(\mathrm{P}=0.18)$. The frequency of epigastric pain was $9.5 \%(6)$ in the children with and $3.8 \%(4)$ in the children without diabetes $(P=0.17)$.

The frequency of epigastric pain was $29.4 \%$ (5) in "the patients with diabetes" with and $2.2 \%$ [1] in those without H.pylori infection $(P=0.004)$.

\section{Discussion}

In the present study, we report no evidence of a relationship between $H$. pylori infection and T1DM in children, and $H$. pylori infection has no effect on glycemic control in children with diabetes, as in agreement with the results of some other studies [13, 14, 19, 20]. Unlike the present study, many studies have suggested that the prevalence of $H$. pylori infection is higher in children with diabetes, compared to children without diabetes [16, 17]; it is therefore still a controversy whether $H$. pylori infection is potentially linked to DM in aspects such as glycemic control, gastrointestinal symptoms, prevalence of infection, eradication rate and reinfection rate [20]. The possible reasons for the disparity observed in the prevalence of infection include differences in study populations in terms of age and sample size. Age should be considered as a factor affecting $H$. pylori infection, as the exposure to $H$. pylori organisms increases with age $[8,14]$, and it is imperative to compare similar age groups when studying the 
prevalence of $H$. pylori infection. In addition, $H$. pylori infection has been diagnosed in different studies using different methods, such as the Urea Breath Test (UBT), the measurement of anti H.pylori IgA, IgG and IgM or H. pylori antigen in the stool and anti Cag A IgG; these different methods justify the disparate findings on this subject. The Present study evaluated fecal $H$. pylori antigens, which may be more relevant than the measurement of $H$. pylori antibody in the serum for active gastrointestinal infection in select patients with diabetes; also the serologic method cannot differentiate between recent and past infections.

Based on the results of some studies, many factors can influence the prevalence of $H$. pylori infection among children with Diabetes, such as age, age at the onset of diabetes, duration of diabetes, BMI, FPG and $\mathrm{HbA}_{1} \mathrm{c} 1[10,16]$. In one study, Feyad et al. Found that children with diabetes and positive H. pylori infection comorbidity are older, have had an earlier onset of diabetes, have a larger BMI and a higher $\mathrm{HbA}_{1} \mathrm{c}$ compared to the negative infected group [10].

Zekry et al. also found that children with diabetes with H. pylori infection have lived with diabetes foe a longer duration of time and have higher insulin dose and $\mathrm{HbA}_{1} \mathrm{c}$; they observed a linear relationship between anti-H pylori IgG titers and $\mathrm{HbA}_{1} \mathrm{c}$ [16]. According to some previous studies, screening for H. pylori infection should be performed in patients with poor metabolic control [1]. One of the possible mechanisms of the association between diabetes and $H$. pylori is that many chemical changes occur following non-enzymatic glycosylation in the stomach that increase sialic acid production, which acts as a receptor for $H$. pylori in the cell surface to attach to the stomach cells [21]. moreover, given the impaired immune response in diabetes, which changes the hormonal and cellular immunity, and also the high prevalence of upper gastrointestinal symptoms in this disease, $H$. pylori may be correlated with diabetes. The findings of the noted study [21], however, are in contrast with the present findings. The present and other researchers [22-24] have found that children with diabetes with and without $H$. pylori infection do not differ in terms of glycemic control.

In this study, the incidence of epigastric pain was significantly higher in the children with diabetes and H. pylori infection comorbidity compared to the children without H. pylori infection. No evidence could be obtained on the effects of $H$. pylori infection in children with diabetes from the north of Iran. This study has examined the relationship between $H$. pylori infection and T1DM for the first time in this region, and its findings can help design other studies that may strive to overcome the limitations of the present study. One of these limitations was the cross-sectional design of the project; an analytical study can better clarify the exact relationship between $H$. pylori infection and T1DM and its effects on glycemic control. On the other hand, available diagnostic tests for detection of H.pylori don't have 100\% accuracy for final diagnosis. So, two or more tests should be performed [25], which is recommended for further studies. Another limitation of the present study was that we didn't deal to gastrointestinal co-morbidities such as coeliac disease, which is recommended for future studies. Also, some variables such as weight loss, socioeconomic status and household size remained undetected, and considering these variables in future studies can help provide a better interpretation of the subject.

To conclude, this study showed that the frequency of H. pylori infection was not significantly different between the children with diabetes and the control group. Glycemic control was also not different between the children with diabetes and without $H$. pylori infection.Nonetheless, this finding may be influenced by other confounders such as insulin dose, nutrition status and socioeconomic status.

Acknowledgements The authors wish to express their gratitude to the Clinical Research Development Unit of Amirkola Children's Hospital and the Research Council and Non-Communicable Pediatric Diseases Research Center of Babol University of Medical Sciences for their contribution to this project.

Funding This study is part of the doctoral dissertation of Dr. Negar Noushiravan and was supported by a research grant from the NonCommunicable Pediatric Diseases Research Center of Babol University of Medical Sciences (Grant Number: 9339615).

\section{Compliance with ethical standards}

Conflict of interest The Authors have No conflicts of interest to declare.

\section{References}

1. Hjelm K, Mufunda E, Nambozi G, Kemp J. Preparing nurses to face the pandemic of diabetes mellitus: a literature review. JAN. 2003;41:424-34.

2. Maahs DM, West NA, Lawrence JM, Mayer-Davis EJ. Epidemiology of type 1 diabetes. Endocrinol Metab Clin N Am. 2010;39:481-97.

3. Arslan D, Kendirci M, Kurtoglu S, Kula M. Helicobacter pylori infection in children with insulin dependent diabetes mellitus. J Pediatr Endocrinol Metab. 2000;13:553-6.

4. Giannakis M, Chen SL, Karam SM, Engstrand L, Gordon JI. Helicobacter pylori evolution during progression from chronic atrophic gastritis to gastric cancer and its impact on gastric stem cells. Proc Natl Acad Sci USA. 2008;105:4358-63.

5. Kusters JG, van Vliet AH, Kuipers EJ. Pathogenesis of helicobacter pylori infection. Clin Microbiol Rev. 2006;19:449-90.

6. Kadhim G, Omar H, Ismail A. Risk factors associated with peptic ulcer disease. J Bioengineer Biomedical Sci. 2015;5:1.

7. Abu-Median HAK, Mirghani UA. Effect of H.pylori on iron deficiency anemia. Khartoum Med J. 2010;30(1):393-7.

8. Nouraie M, Latifi Navid S, Rezvan H, Radmard AR, Maghsudlu $\mathrm{M}$, Zaer-Rezaii $\mathrm{H}$, et al. Childhood hygienic practice and family education status determine the prevalence of helicobacter pylori infection in Iran. Helicobacter. 2009;14:40-6.

9. Chiu W, Chick W, Kwok K. A home-made rapid urease test in the diagnosis of helicobacter pylori infection. Singap Med J. 1999;40: 243-5. 
10. Fayed SB, Dayem E, Abd SM, Khalil E, El Kader MA, El Halim EA. Helicobacter pylori infection in children with type 1 diabetes mellitus. Maced J Med Sci. 2014;7:114-8.

11. Papamichael KX, Papaioannou G, Karga H, Roussos A, Mantzaris GJ. Helicobacter pylori infection and endocrine disorders: is there a link. World J Gastroenterol. 2009;15:2701-7.

12. Marietti M, Gasbarrini A, Saracco G, Pellicano R. Helicobacter pylori infection and diabetes mellitus: the 2013 state of art. Panminerva Med. 2013;55:277-81.

13. Zhou X, Zhang C, Wu J, Zhang G. Association between helicobacter pylori infection and diabetes mellitus: a metaanalysis of observational studies. Diabetes Res Clin Pract. 2013;99:200-8.

14. Bazmamoun H, Rafeey M, Nikpouri M, Ghergherehchi R. Helicobacter pylori infection in children with type 1 diabetes mellitus: a case-control study. J Res Health Sci. 2016;16:68-71.

15. Begue RE, Gomez R, Compton T, Vargas A. Effect of helicobacter pylori eradication in the glycemia of children with type 1 diabetes: a preliminary study. South Med J. 2002;95:842-6.

16. Zekry OA, Elwahid HAA. The association between helicobacter pylori infection, type 1 diabetes mellitus, and autoimmune thyroiditis. J Egypt Public Health Assoc. 2013;88:143-7.

17. Gasbarrini A, Ojetti V, Pitocco D, De Luca A, Franceschi F, Candelli M, et al. Helicobacter pylori infection in patients affected by insulin-dependent diabetes mellitus. Eur J Gastroenterol Hepatol. 1998;10:469-72.

18. Woodward M, Morrison C, Mccoll K. An investigation into factors associated with helicobacter pylori infection. J Clin Epidemiol. $2000 ; 53: 175-81$.
19. Xia HH, Talley NJ, Kam EP, Young LJ, Hammer J, Horowitz M. Helicobacter pylori infection is not associated with diabetes mellitus, nor with upper gastrointestinal symptoms in diabetes mellitus. Am J Gastroenterol. 2001;96:1039-46.

20. El-Eshmawy MM, El-Hawary AK, Abdel Gawad SS, El-Baiomy AA. Helicobacter pylori infection might be responsible for the interconnection between type 1 diabetes and autoimmune thyroiditis. Diabetol Metab Syndr. 2011;3(1):28.

21. Valkonen KH, Ringner M, Ljungh $\AA$, Wadström T. High-affinity binding of laminin by helicobacter pylori: evidence for a lectin-like interaction. FEMS Immunol Med Microbiol. 1993;7:29-37.

22. Dai Y-N, Yu W-L, Zhu H-T, Ding J-X, Yu C-H, Li Y-M. Is helicobacter pylori infection associated with glycemic control in diabetics? World J Gastroenterol. 2015;21:5407-16.

23. Chobot A, Bak-Drabik K, Skała-Zamorowska E, Krzywicka A, Kwiecien J, Polanska J. Helicobacter pylori infection in type 1 diabetes children and adolescents using $13 \mathrm{C}$ urea breath test. Pol J Microbiol. 2014;63:63-7.

24. Horikawa C, Kodama S, Fujihara K, Yachi Y, Tanaka S, Suzuki A, et al. Association of Helicobacter pylori infection with glycemic control in patients with diabetes: a meta-analysis. J Diabetes Res. 2014.

25. Okuda M, Lin Y, Kikuchi S. Helicobacter pylori infection in children and adolescents. Adv Exp Med Biol. 2019;1149:107-20.

Publisher's note Springer Nature remains neutral with regard to jurisdictional claims in published maps and institutional affiliations. 